\title{
The Adding Role and Accuracy of Chest Ultrasound and Doppler in Peripheral Lung Lesions
}

\author{
YOUSSRIAH Y. SABRI, M.D.*; DINA A.A. MUHAMMED, M.D.*; KHALED M. HELMI EL KAFFAS, M.D.*; \\ HEBA ALLAH H. ASSAL, M.D.**; MONA A. FOUAD HAFEZ, M.D.* and SHADY N. MASHHOUR, M.D.* \\ The Department of Diagnostic \& Interventional Radiology* and Chest Department**, Cairo University School of Medicine, \\ Kasr Al-Ainy Hospital, Cairo, Egypt
}

\begin{abstract}
Background: Chest ultrasound (US) and color Doppler sonography play important roles in the diagnosis and characterization of different pathologies of peripheral lung lesions, especially in the emergency setting.
\end{abstract}

Aim of Study: The aim of this study is to assess the role of chest US and Doppler examination in the assessment of peripheral lung lesions in comparison with chest $\mathrm{CT}$, which is the gold standard for chest diagnosis.

Subjects and Methods: This cross-sectional study involved 120 patients who underwent chest CT examination, full chest US, and Doppler. Results were assessed blindly and compared with CT.

Results: The main lung lesions assessed by US and Doppler were lung collapse, consolidation, interstitial lung changes, and peripheral pulmonary nodules, with the estimated sensitivity, specificity, positive predictive value, negative predictive value, and accuracy of detection ranging from $63.9 \%-76.9 \%$, $94 \%-95 \%, 79.2 \%-95 \%, 85.9 \%-91.5 \%$, and $85 \%-93.3 \%$ respectively. There was a significant correlation between US and Doppler in comparison with CT.

Conclusion: Chest US proved its value as a complementary tool for the diagnosis of peripheral lung lesions, with high specificity and accuracy. This finding was enforced by Doppler, history, and clinical suspicion, although the use of CT to obtain the final diagnosis would be more applicable.

Key Words: Chest ultrasound - Chest Doppler-Chest CTPeripheral lung lesion.

\section{Introduction}

TRANSTHORACIC ultrasound (US) has become an important noninvasive, readily available, and bedside diagnostic tool. It can complement imaging evaluation, physical examination, and clinical evaluation [1].

Correspondence to: Dr. Shady N. Mashhour, E-Mail: shady.mashhour@kasralainy.edu.eg
Chest US plays an important role in the diagnosis of different pathologies, which depends mainly on the variations of US artifacts. The chest wall, pleura, and peripheral pulmonary parenchyma are the main sites visualized by US. The role of US in the diagnosis of lung lesions is limited to peripheral lesions and is still a controversial subject of research $[2,3]$.

Color Doppler sonography is a dynamic and reliable method that can characterize the vascularization of peripheral pulmonary lesions as well as their flow signal patterns. It is used to differentiate pleural from pulmonary parenchymal lesions [4], which is helpful for differentiating lung cancers from benign lesions, assessing the neovascularity of lung cancers, diagnosing congenital vascular abnormalities (pulmonary sequestration and arteriovenous malformation), and using US-guided needle biopsy avoids the complication of injury to the great vessels [5]

Peripheral lung consolidation is seen as a subpleural hypoechoic region or tissue-like echotexturethat moves with respiration. Air or fluid bronchograms may be seen and appear as branching hyperechoic lines arising from the hilum that move with respiration $[\mathbf{6 , 7 , 8 ]}$. The effect on the sonographic diagnosis of interstitial tissues depends on the visualization of some vertical reverberation artifacts, the B-lines, which are caused by a close

\footnotetext{
Abbreviations:

CDUS : Color Doppler sonography.

DPLD : Diffuse parenchymal lung disease.

ILD : Interstitial lung disease.

PE : Pulmonary embolism.

TUS : Transthoracic ultrasound.
} 
interface between the alveolar air and the increased interstitial fluids [9].

Obstructive atelectasis is marked by a homogeneous, hypoechoic presentation of the lung tissue, as in hepatization. Effusion is absent or very mild. In cases of lobar atelectasis, there is a rather distinct margin toward the ventilated lung tissue. Depending on the duration of atelectasis, intraparenchymatous structures also may be seen as hypoechoic vascular lines and echogenic reflexes or anechoic, hypoechoic, or echogenic focal lesions [10]

Peripheral lung tumors are seen as homogeneous, well-defined hypoechoic or echogenic masses showing posterior acoustic enhancement. The pleural line may be interrupted if the tumor extends to the pleura and the extension of the chest wall is detected $[\mathbf{8 , 1 1 , 1 2 ]}$.

In this study, we aimed to compare the use of CT of the chest, which is the gold standard for chest diagnosis, with chest US and Doppler examination for the assessment of peripheral lung lesions and to study their added value.

\section{Subjects and Methods}

The patients were referred from the chest department and chest outpatient clinic to the radiology department between July 2017 and August 2019. The research protocol was approved by the Institutional Human Research Ethics Committee. Written informed consent was obtained from the participantsor from their parents in the pediatric age group.

This cross-sectional study involved 120 patients, including 60 females and 60 males, ranging in age 5 to 88 years.

All patients were subjected to a clinical examination with history taking and relevant laboratory tests. Pulmonary function tests were also administered to patients with suspected interstitial pulmonary fibrosis.

The criterion for study inclusion was the presence of peripherally located lung lesions detected on $\mathrm{CT}$ of the chest. Exclusion criteria were normal lungs detected on US and chest CT, lesions other than peripheral lung lesions as pleural lesions only, uncooperative cases, and patients with poor US image quality.

All patients underwent chest US using a GE LOGIQ US machine. Two radiologists with 6 to 15 years of experience in chest US who were blinded to patient details evaluated the $\mathrm{CT}$ of each patient over a time interval of 0-2 days.

Probes:

We used a high-resolution 5- to $10-\mathrm{MHz}$ linear transducer to image the thoracic wall and the parietal pleura, and a convex $3-$ to $5-\mathrm{MHz}$ transducer to image for lung parenchyma.

\section{Technique:}

Most patients were examined in supine, right lateral, left lateral, and sitting positions. Patients with orthopnea or respiratory distress (16 patients, $13.3 \%$ ) were examined only in the upright and sitting positions. Patients who presented with disturbed conscious level or bed ridden patients (7 patients, 5.8\%) were examined only in the supine position.

The convex probe was placed subcostally and longitudinally from the right for transhepatic examination, with slight tilting in the cranial direction. The liver, hepatic veins, diaphragm, and reflection of the liver were revealed to be above the diaphragm and the lung curtain sign. The same technique performed on the left subcostal aspect revealed that the spleen, diaphragm, and reflection of the spleen were above the diaphragm and the lung curtain sign. Moving the probe in an upward direction allowed for screening of the pleural recesses for effusion.

The convex probe was placed longitudinally vertical to the ribs, revealing the intercostal muscles, two ribs with their acoustic shadows, the intervening intercostal space showing the pleura, and underlying lung parenchyma. The convex probe was placed parallel to the ribs in the intercostal space, which revealed the intercostal muscles, pleura, and underlying lung parenchyma. This was done in both lung apices to exclude pneumothorax by observing pleural sliding and B-lines in addition to the use of M-mode.

If the patient was alert and complained of localized chest pain (25 patients, $20.8 \%$ ), a focused chest US was performed at the site of the pain.

The linear probe placed parallel to the ribs in the intercostal space revealed the intercostal space, muscle, and pleura. The linear probe placed parallel to the ribsrevealed the ribs as a hyperechoic surface with prominent acoustic shadows beneath the ribs. The linear probe placed vertically to the ribs revealed the ribs with their acoustic shadows and intervening intercostal space, muscle, and the pleura, called the "bat-wing sign". 
Transthoracic Doppler US was performed for all patients. The color Doppler and spectral wave Doppler were assessed for all lesions. However, in patients with severe dyspnea and respiratory distress (32 patients, 26.6\%), the Doppler was not conclusive because of uncontrollable respiratory movements and the inability of patient to hold their breath for proper examination.

All patients underwent $\mathrm{CT}$ of the chest using different techniques with a 16-slice Siemens MSCT scanner SOMATOM machine: $53.3 \%$ had chest CT without contrast, $23.3 \%$ post contrast, $23.3 \%$ CT pulmonary angiography, and $3.3 \%$ highresolution CT (HRCT), according to the suspected clinical diagnosis. CT scans were evaluated by another two radiologists with 14 and 30 years of experience. Results of US, Doppler, and chest CT were then compared.

Statistical analysis was conducted using the following procedures. Data were coded and entered using the Statistical Package for the Social Sciences version 25. Data were summarized using mean, standard deviation, minimum, and maximum for quantitative data and using frequency (count) and relative frequency (percentage) for categorical data. Standard diagnostic indices including sensitivity, specificity, positive predictive value, negative predictive value, and diagnostic efficacy were calculated.

To compare categorical data, we performed a chi-square test. When the expected frequency was less than 5, Fisher exact test was used instead. A $p$-value $<0.05$ was considered statistically significant.

\section{Results}

A total of 120 patients were included in this study (60 females and 60 males, age range 5-88 years, average age 43.69 years [SD 19.94 years]). The patients' main presentations were dyspnea in $47.5 \%$ of cases, chest pain in $22.5 \%$ of cases, cough in $28.3 \%$ of cases, hemoptysis in $2.5 \%$ of cases, and fever in $10.8 \%$ of cases.

Chest CT showed variable peripheral pulmonary lesions, with incidental associated extrapulmonary pleural lesions (effusion in 46 cases, pneumothorax in 10 cases, thickening in 11 cases, and irregularity in 9 cases), bone lesions (rib fracture in 6 cases, an osteolytic lesion in 1 case), and chest wall lesions (chest wall hematoma in 1 case and surgical emphysema in 5 cases), as displayed in Table (1).
US detected consolidative lesions with different etiologies, including pneumonia in 14 cases (Fig. 1) and contusion in 6 cases (Fig. 2). CT pulmonary angiography detected 4 patients with findings of pulmonary embolism (PE) and infarctions, all of which appeared as peripheral consolidative lesions by US (Fig. 3) with associated localized or free effusion and absent vascularity by Doppler examination confirming pulmonary infarction.

Lung collapse was detected in $28.3 \%$ of patients by US as compared with $32.5 \%$ by chest CT. US was able to reveal a minimal alveolar filling process and atelectatic changes, even in cases of minimal effusion (Figs. 2,4).

Interstitial tissue affection or interstitial syndrome was detected on US as nonspecific findings, B-lines (in 61 patients, 50.8\%), and increased Alines or reverberation artifacts (60 patients, $50 \%$ ) (Fig. 5). The distribution of B-lines, being multiple diffuse and with bilateral mounting to lung rockets based on the degree of fibrosis, and the associated pleural lesions as pleural thickening $>3 \mathrm{~mm}$ ( 23 patients, 19.2\%) and irregularities (51 patients, $42.5 \%$ ) may differentiate between these conditions. B-lines were detected in 61 patients (50.8\%); 43 patients had interstitial lung fibrosis or parenchymal scarring (focal or diffuse), 14 patients had interstitial pulmonary edema either due to generalized anasarca or heart failure, 1 patient had a pulmonary hemorrhage, and finally, 3 patients had pneumonia.

The use of US for the detection of peripheral pulmonary nodules or masses (Fig. 6) revealed that $23.3 \%$ of patients had peripheral pulmonary nodules or masses, whereas this value was $30 \%$ using CT. The assessment of the surrounding parenchyma and associated findings added to the value of US and limited the differential diagnosis as overlying pleural sliding, diaphragmatic mobility, and Doppler examination of the nodules or masses.

The following associated findings were detected by US: Chest wall lesions (such as rib fracture in $4.2 \%$ of cases, chest wall hematoma in $1.7 \%$ of cases, and surgical emphysema in $4.2 \%$ of cases) and pleural lesions (such as pleural collection in $46.7 \%$, pneumothorax in $7.5 \%$, pleural thickening in $19.2 \%$, and pleural irregularity in $42.5 \%$ ).

Chest CT is considered the gold standard for thoracic imaging. Statistical data (sensitivity, specificity, positive and negative predictive value, as well as accuracy) were obtained by comparing US with chest CT (Table 2), which indicated a strong significant correlation and $p<.00001$. 
Table (1): Main CT findings/lesions in the studied patients.

\begin{tabular}{llc}
\hline & Lesions detected by CT & No. (\%) of patients \\
\hline Peripheral pulmonary lesions & Pneumonia & $19(15.8 \%)$ \\
& Lung infarction & $4(3.3 \%)$ \\
& Lung contusion/laceration & $10(8.3 \%)$ \\
& Lung nodule/mass & $36(30.0 \%)$ \\
& Lung collapse & $39(32.5 \%)$ \\
& Ground-glass opacities & $31(25.8 \%)$ \\
Associated bone, chest wall, and pleural findings & Honeycombing & $6(5.0 \%)$ \\
& Interstitial lung disease & $29(24.2 \%)$ \\
& Bone lesions & $7(5.8 \%)$ \\
& Chest wall lesions & $6(5 \%)$ \\
& Pleural lesions & $76(63.3 \%)$ \\
\hline
\end{tabular}

Table (2): Statistical indices of different peripheral lung lesions detected by chest ultrasound in comparison with CT.

\begin{tabular}{lccccccc}
\hline Lesion & CT & US & Sensitivity & Specificity & $\begin{array}{c}\text { Positive } \\
\text { predictive value }\end{array}$ & $\begin{array}{c}\text { Negative } \\
\text { predictive value }\end{array}$ & Accuracy \\
\hline Lung collapse & $39(32.5 \%)$ & $34(28.3 \%)$ & $76.9 \%$ & $95.06 \%$ & $88.24 \%$ & $89.53 \%$ & $89.17 \%$ \\
Consolidation & $33(27.5 \%)$ & $24(20 \%)$ & $67.8 \%$ & $94.57 \%$ & $79.17 \%$ & $90.62 \%$ & $88.33 \%$ \\
Interstitial lung changes & $66(55 \%)$ & $61(50.8 \%)$ & $92.0 \%$ & $94.74 \%$ & $95.08 \%$ & $91.53 \%$ & $93.33 \%$ \\
Pulmonary nodule/mass & $36(30 \%)$ & $28(23.3 \%)$ & $63.8 \%$ & $94.05 \%$ & $82.14 \%$ & $85.87 \%$ & $85.00 \%$ \\
\hline
\end{tabular}
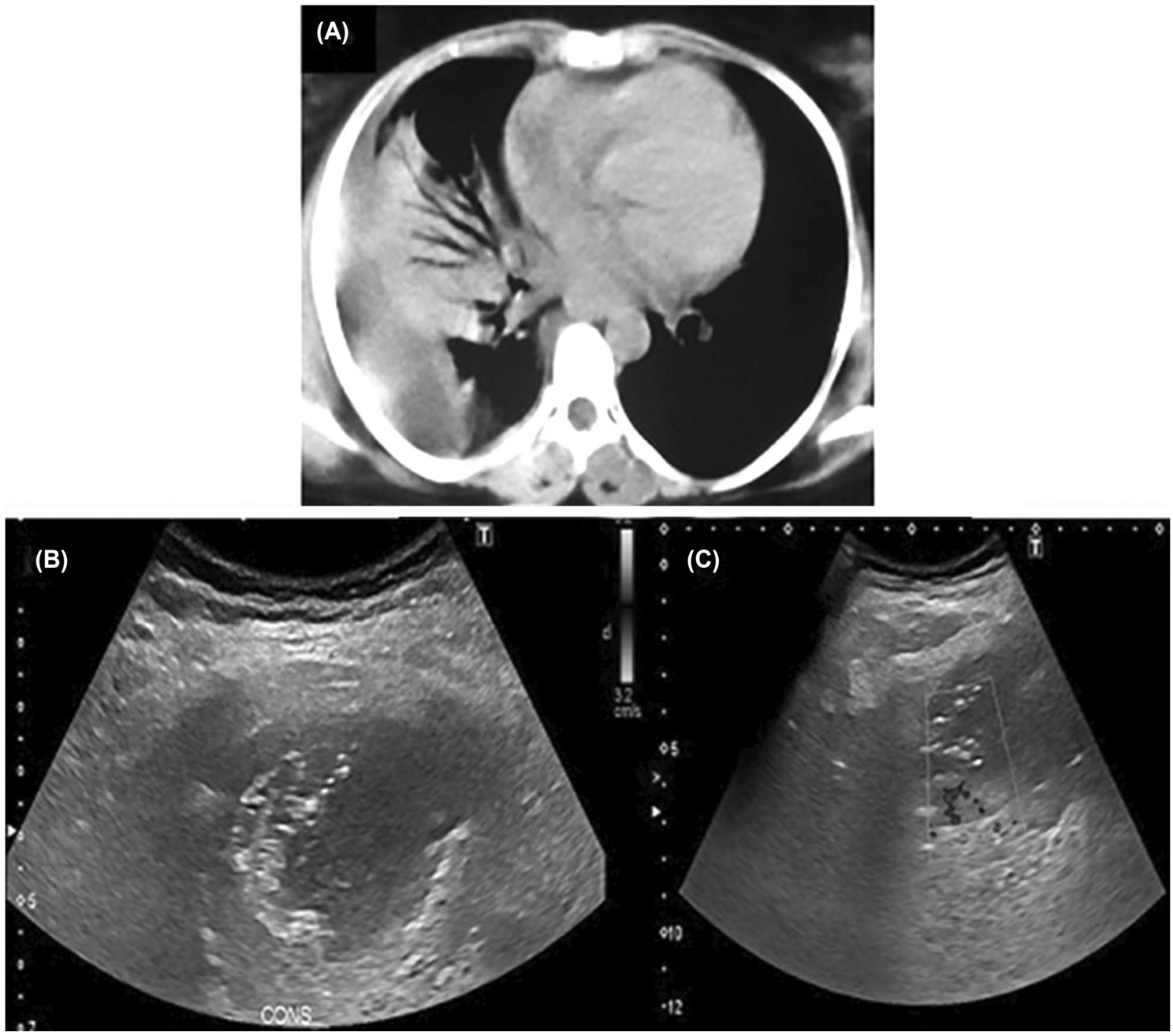

Fig. (1): Thirty-six-year-old woman who presented with fever and acute right-sided chest pain of 1-week duration. (A) The axial CT mediastinal window showed right middle lobe lateral segment consolidation and mild right-sided partially encysted pleural effusion. (B, C) Chest US and Doppler showed right lateral mid zonal consolidation with air bronchogram, with relatively crowded internal bronchi, witha normal pulmonary treelike vascularity with relatively crowded vessels. Diagnosed as pneumonia with parapneumonic effusion. 

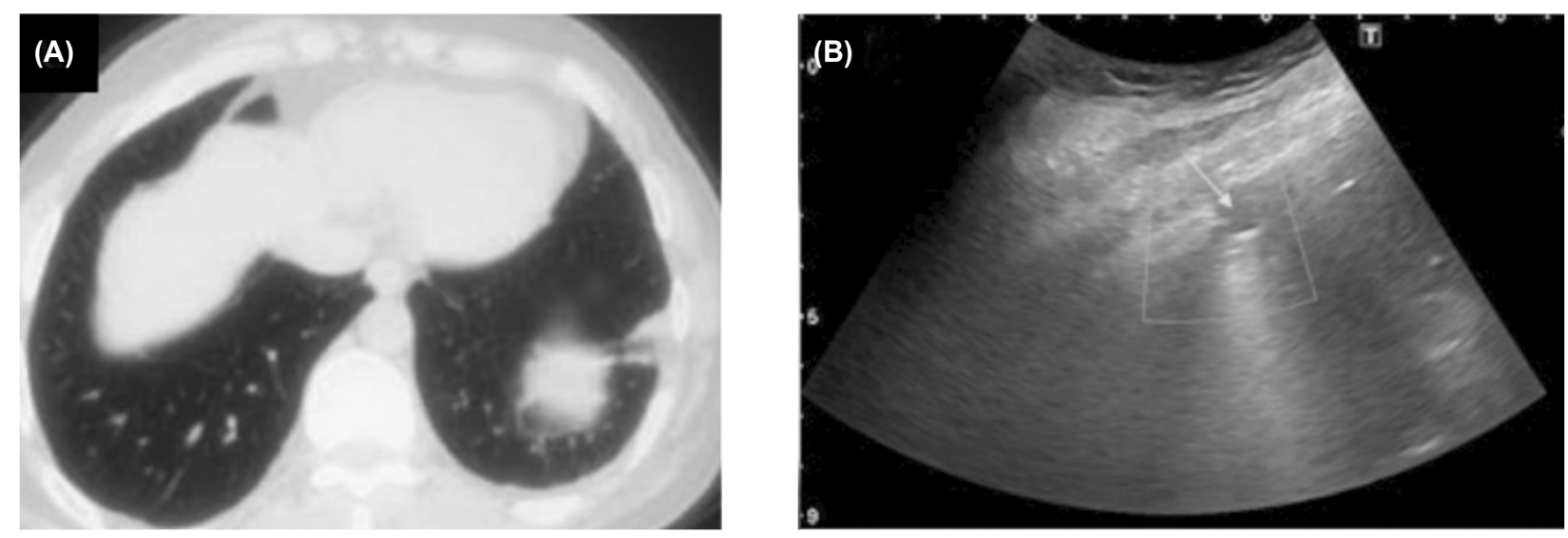

Fig. (2): Forty-two-year old man with a history of chest trauma and rib fractures. (A) Chest CT lung window showed left lower lobe lateral basal segments with well-defined peripheral lesion reflecting residual lung lesion. (B) Chest US and Doppler revealed left lateral lower zonal; well-defined hypoechoicsubpleuralis seen showing no vascularity by Doppler examination, diagnosed as pulmonary contusion/laceration.
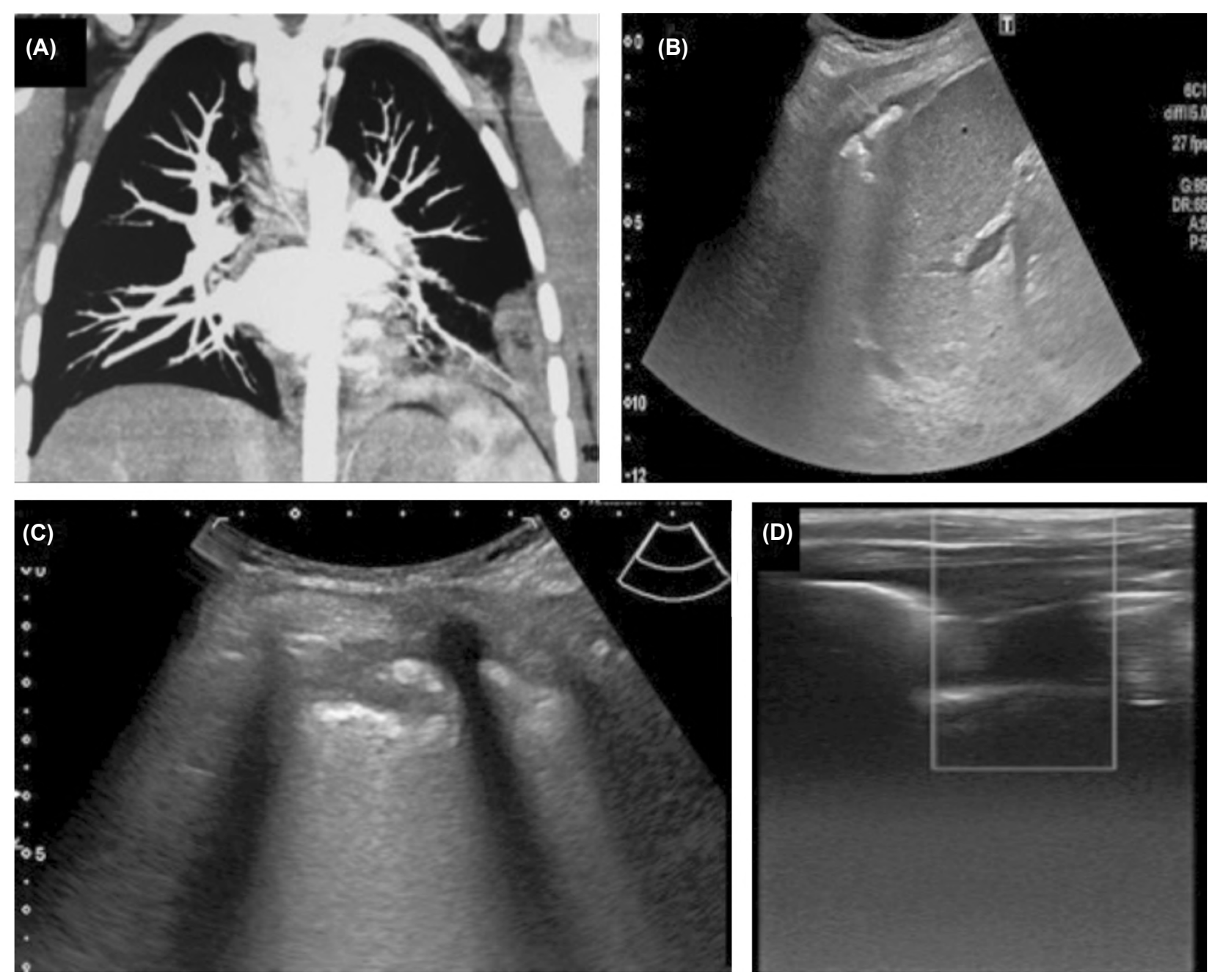

Fig. (3): Fifteen-year-old man who complained of acute dyspnea with localized left-sided chest pain and hemoptysis. (A) Chest CT coronal image mediastinal window showed left lower lobe lateral segment subpleural wedge-shaped consolidation and mild left pleural effusion. (B, C, D) Chest US and Doppler revealed mild left pleural effusion with minimal compression collapse underneath (arrow,B). Left basal subpleural fairly round hypoechoic area (arrow,C) showed no vascularization by Doppler study (D). Diagnosed as pulmonary infarction. 


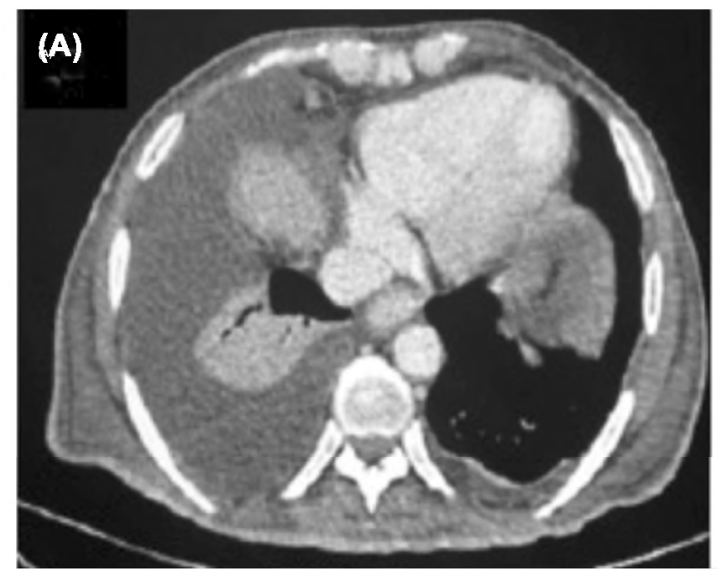

Fig. (4): Sixty-four-year-old man who presented with obstructive jaundice, hepatosplenomegaly, and ascites who developed bilateral pleural effusion. (A) Chest CT of the mediastinal window showed moderate right pleural effusion with lower lobe relaxation collapseunderneath andmild left pleural effusion. $(B, C)$ Chest ultrasound revealed moderate right pleural effusion with lower lobe relaxation collapseunderneath (B, arrow). Multiple bilateral B-lines and lung rockets, denoting interstitial affection likely edema (arrow, C).
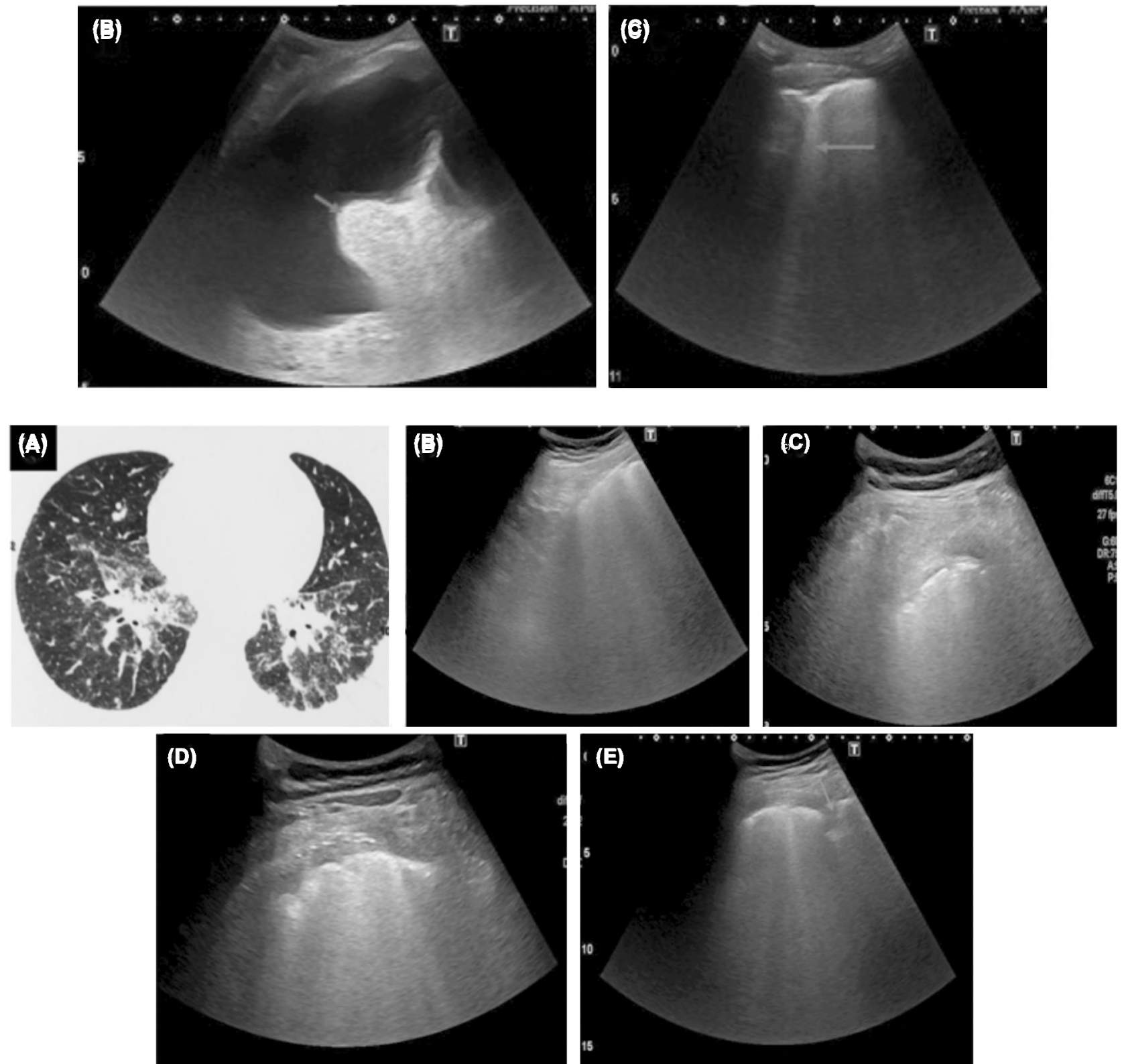

Fig. (5): Forty-year-old woman diagnosed with chronic sarcoidosis on medical treatment. She complained of progressive dyspnea and dry cough. (A) HRCT showedbilateral patchy ground-glass opacities, few dispersed thickened interlobular septa, prominent bronchocentricinterstitium, and dispersed, variable-size nodules with beaded fissures. (B-D) Chest ultrasound revealedbilateral multiple diffuse B-lines with an average distance of $7 \mathrm{~mm}$ (B7) (B). Increased reverberation artifacts (A-lines) (C, arrow). Bilateral pleural surface irregularities and pleural thickening (D). Bilateral lower lobar scattered subpleural nodules (E, arrow). 

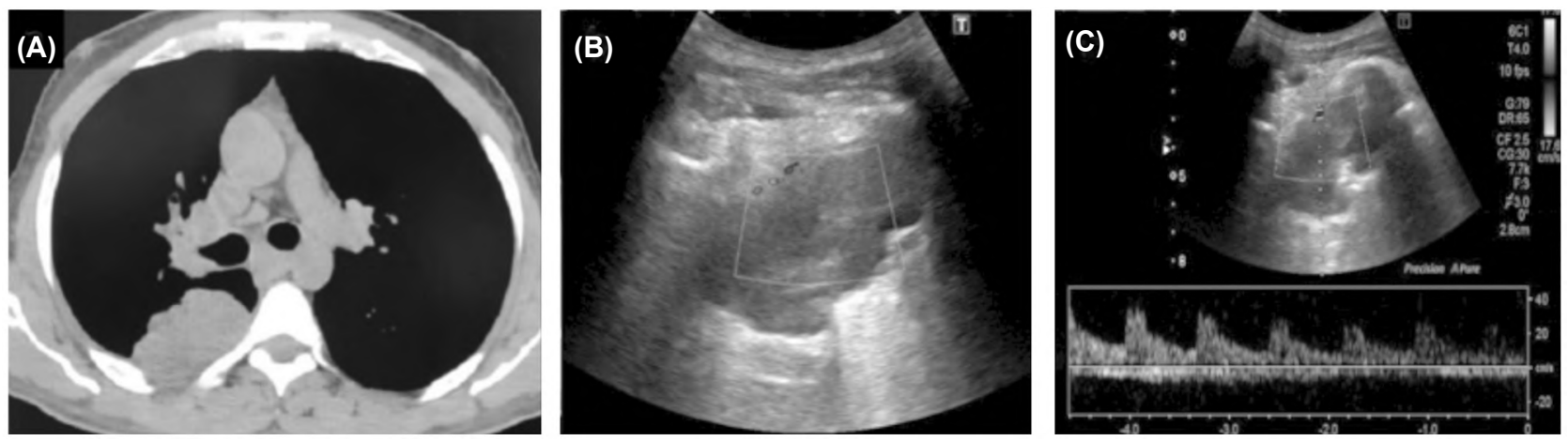

Fig. (6): Thirty-eight-year-old man who complained of right upper-back pain for 1 month. (A) Chest CT mediastinal window showed a right lower lobe apical segment paravertebral mass with parahilar extension associated with right hilar and mediastinal lymphadenopathy, suggestive of malignant mass. (B, C) Chest US and Doppler revealed a well-defined hypoechoic pleuralbased lesion at the right upper posterior back, showing heterogeneous echogenicity (B). The hypoechoicsubpleural lesion showed irregular internal and peripheral pleural-derived vasculature showing arterial triphasic flow waves (C). An ultrasound-guided biopsy was obtained from the lesion, and histopathological examination revealed adenocarcinoma of the lung.

\section{Discussion}

US is safe, nonionizing, effective, and affordable. Ultrasonic examination of the chest is a rapidly developing application that can be used to evaluate a wide range of peripheral, parenchymal, pleural, and chest wall diseases. The technique is suitable for bedside use in the intensive care unit, where suboptimal radiography may mask or mimic clinically significant lesions that are difficult to differentiate between pleural and parenchymal changes [13].Furthermore, US is increasingly used to guide interventional procedures of the chest, such as biopsy and placement of intercostal chest drains

The purpose of this study was to assess the role of US in the evaluation of peripheral pulmonary lesions as compared with chest CT. In presence of an acoustic window or pleural-based lesion, lung US can detect, describe, and delineate peripheral pulmonary lesions. In addition, Doppler examination of such lesions would narrow the differential diagnosis.

In this study, lung collapse was detected in $28.3 \%$ of patients by US as compared with $32.5 \%$ by chest CT. US was able to reveal minimal alveolar filling process and atelectatic changes, even in cases of minimal effusion. Its sensitivity was $63.8 \%$ and specificity was $94 \%$, and the results were comparable with those of Sabri et al., [15], whose study on US of the chest in in patients with chest trauma showed a calculated sensitivity of $68.4 \%$ and sensitivity of $98.8 \%$.

Thirty-six patients (30\%) in this study were diagnosed with pulmonary nodules or masses, 23 of which were detected by US (23.3\%). Among the studied patients, 9 patients were proven to have metastatic nodules, and 5 patients were pathologically proven to have primary malignant tumors, such as neuroendocrine tumors and adenocarcinoma of the lungs. Nine patients had nonspecific nodules associated with interstitial lung disease (ILD), and 13 patients had pulmonary nodules associated with inflammatory or granulomatous lung disease. Clinical data, including history of malignant process, chronic granulomatous infection, or signs of acute infection, were confirmed by Doppler examination of these nodules or masses, which helped to limit the differential diagnosis. The calculated sensitivity was $63.8 \%$, specificity was $94 \%$, and positive and negative predictive values were $82.14 \%$ and $85.87 \%$, respectively. However, in their study, Caroli et al., [8] found sensitivity and specificity values of $88.89 \%$ and $100 \%$, respectively. Furthermore, positive predictive and negative predictive values were $100 \%$ and $93.33 \%$, respectively. The accuracy of thoracic US (TUS) was $95.65 \%$ of the sliding sign for the detection of chest wall infiltration in non-small-cell lung cancer and lung infiltration by chest wall tumors, and they stated that the main advantage of TUS over CT was in providing dynamic, real-time imaging of the system consisting of lung tumor and pleura-chest wall involvement. The US sign of the movement of the two pleural layers is the so-called "sliding sign". The absence of this sign predicts the presence of pleural adhesions.

In the current study, $27 \%$ of patients had pulmonary consolidation as detected by CT, whereas US diagnosed $20 \%$ of cases. However, in the prospective study by Nazerian et al., [16] analyzing 285 patients, the calculated sensitivity and specificity were $67.86 \%$ and $94.57 \%$, respectively. Lung 
US was feasible in all patients with a calculated sensitivity of $82.8 \%$ and a specificity of $95.5 \%$. The sensitivity increased to $91.7 \%$ and the specificity to $97.4 \%$ in patients with complaints of pleuritic chest pain.

In the current study, four patients were diagnosed with pulmonary infarction (3.3\% of cases). All a for ementioned patients were diagnosed with pulmonary infarctionby US powered by Doppler study. This infarction appeared sonographically as a well-defined oval or triangular pleural-based hypoechoic area with absent vascularity by Doppler examination. CT pulmonary angiography showed wedge-shaped pleural-based consolidative lesions, two of which showed pulmonary vascular filling defects, whereas the other two patients showed chronic thromboembolic findings with pulmonary hypertension. In comparison, Reissig et al., [17], in their prospective study of 69 patients with suspected $\mathrm{PE}$,made a diagnosis of PE in 44 patients. Ninety-one peripheral parenchymal lesions associated with PE were detected by TUS in 35 patients $(80 \%)$. They showed multiple, triangular, hypoechoic, and pleural-based parenchymal lesions with a localized and/or basal effusion. Those authors found that TUS had a sensitivity of $80 \%$ and specificity of $92 \%$ for detecting PEs, with positive and negative predictive values of $95 \%$ and $72 \%$, respectively, and an accuracy of $84 \%$.

In this study, $15.8 \%$ of patients were diagnosed with pneumonia by chest CT, and $11.6 \%$ of patients were diagnosed by US. These patients showed either lobar or segmental hypoechoic areas with preserved or mildly reduced pulmonary vascularity by the Doppler study. Associated air bronchogram, as well as localized or free pleural effusion, were detected with the aid of clinical data, with a calculated sensitivity of $73.7 \%$, specificity of $100 \%$, positive predictive value of $100 \%$, negative predictive value of $95.3 \%$, and accuracy of $95.8 \%$. Abdel Kader et al., [4] found an opacity with air bronchogram in all lesions on chest CT (100\%); in the diagnosis of pneumonia complicated by effusion, lung US with color Doppler showed high sensitivity (100\%) and specificity (93.8\%), and the comparable diagnostic accuracy was $96.8 \%$. These results are in near agreement with the CT findings. Xia et al., [18] reported that the calculated sensitivity and specificity of TUS in cases with pneumonia were $90.9 \%$ and $89.7 \%$, respectively, and the combination of chest imaging and clinical criteria increased the sensitivity to $95 \%$ and the specificity to $91.3 \%$.
Interstitial tissue affection or interstitial syndrome was detected on US as nonspecific findings, with cases of pulmonary fibrosis, interstitial lung infections, heart failure, and acute respiratory distress syndromeshowing a similar sonographic pattern. In this study, B-line artifacts were found in $50.8 \%$ of patients, indicating interstitial tissue disease. They were categorized as B0 (absent) in $49.1 \%$ of patients; as B3 (with a distance of $3 \mathrm{~mm}$ ), which is a less severe form,in $34.1 \%$ of patients; and as B7 artifacts (with distance of $7 \mathrm{~mm}$ ), which is a more frequent and severe form, in $16.6 \%$ of patients. The calculated sensitivity and specificity were $92.1 \%$ and $94.7 \%$, respectively. In cases of ILD, B3 artifacts corresponded to ground-glass opacities in HRCT of the lung, whereas B7 corresponded to honeycombing and/or advanced fibrosis. B7 may also be seen in cases of interstitial pulmonary edema. Dirty shadowing or fused B-lines created the lung rockets, which were described in advanced fibrosis or severe forms of pulmonary edema, pulmonary hemorrhage, or respiratory distress. Associated US findings in ILDs included pleural changes, such as pleural thickening and interruption of the pleural surface, giving pleural irregularities.

Dubinsky et al., [19] studied the presence of the B-line (grade 1) and reported a sensitivity of $79 \%$ and specificity of $58 \%$. In contrast, the presence of a grade $3 \mathrm{~B}$-line had low sensitivity (33\%) but was highly specific for diffuse parenchymal lung disease (DPLD; 100\%). The presence of a B-line of grade 2 or greater was moderately sensitive $(62 \%)$ but also had high specificity at $92 \%$. The severe form of B-lines is truly specific for the presence of DPLD. The more severe the lung disease, the greater the number and depth of the B-lines, as larger numbers of lymphatics are distended to a greater extent in the presence of DPLD, thus creating more and larger B-lines, and this was correlated with the presence of DPLD, especially fluid overload, lung edema, and elevated wedge pressures [19].

In this study, Doppler examination revealed a preserved tree like pulmonary vascularity in $75.8 \%$ of cases diagnosed as consolidation and atelectasis. Abnormal single dispersed intralesional vascularity was found in neoplastic sizable pulmonary nodules or masses (14 patients, 11.6\%): 9 patients had proven metastatic pulmonary nodules, and 5 patients had pathologically proven primary pulmonary malignant tumors. Absent vascularity was detected in cases of pulmonary infarction (4 patients, 3.3\%) or end-stage fibrosis (11 patients, $9.1 \%$ ). 
In their study, Gorg et al., [20] reported thatno vascular signals were found by CDS mapping, including a case with a scar, patients with pulmonary infarcts, and a patient with peripherally based lung cancer.

On the other hand, a vascular tree or marked vascularity was found in patients who had more malignant than benign lesions, with significant differences between benign and malignant lesions $(p=.005)$ and between small and large lesions $(p=.05)$ regarding vascularity, and there was no significant difference in the number of flow signals between benign and malignant lesions.

The clinical examination, history, and associated findings helped to limit the differential diagnosis for each case. The role of US in the acute settingis growing; however, in this study, we recommend that US with the aid of Doppler could be used as a complementary technique to CT for the assessment of the nature of peripheral lung lesions, internal vascularity, and overlying pleural cover and thus could limit the differential diagnosis and drive through a final diagnosis.

The limitations of this study include the use of different chest CT protocols, which were based on the suspected clinical diagnosis, and the broad spectrum of studied diseases incorporating all peripheral lung lesions, as they all have intermingled US findings. Thus, the associated clinical conditions could limit the differential diagnosis.

\section{Conclusion:}

Chest US proved its value as a complementary technique for the diagnosis of peripheral lung lesions with high specificity and accuracy. This value is enforced by Doppler and associated findings, history, and clinical suspicion, while a final diagnosis using $\mathrm{CT}$ would be more applicable.

\section{References}

1- SAYED S. S., AGMY G.M., SAID A.F. and KASEM A.H.: Diagnostic performance of trans-thoracic sonography in patients of pneumonia and pulmonary embolism. Egypt J. Chest Dis. Tuberc., 65: 621-628, 2016.

2- ALKHAYAT K.F. and ALAM-ELDEEN M.H.: Value of chest ultrasound in diagnosis of community acquired pneumonia. Egypt J. Chest Dis. Tuberc., 63: 1047-1051, 2014.

3- RIZK A.M., ZIDAN M.A., EMARA D.M., et al.: Chest ultrasound in the assessment of patients in ICU: How can it help? EJRNM, 48: 313-322, 2017.

4- ABDEL KADER M., NASR M. and OSMAN M.: Implementation of chest ultrasound with color Doppler in diagnosis of pneumonia in adults. EJRNM, 47: 771-781, 2016.
5- HSU W., YU Y., TU C., et al.: Color Doppler US pulmonary artery vessel signal: A sign for predicting the benign lesions. Ultrasound Med. Biol., 33: 79-88, 2007.

6- KOEGELENBERG C.F., VON GROOTE-BIDLINGMAIER F. and BOLLIGER C.T.: Transthoracic ultrasonography for the respiratory physician. Respiration, 84: 337-350, 2012.

7- VOLPICELLI G., ELBARBARY M., BLAIVAS M., et al.: International evidence-based recommendations for point-of-care lung ultrasound. Intensive Care Med., 38: 577-591, 2012.

8- CAROLI G., DELL'AMORE A., CASSANELLI N., et al.: Accuracy of transthoracic ultrasound for the prediction of chest wall infiltration by lung cancer and of lung infiltration by chest wall tumours. Heart Lung Circ., 24: 1020-1026, 2015.

9- VOLPICELLI G.: Lung sonography. J. Ultrasound Med., 32: 165-171, 2013.

10- MATHIS G.: Chest Sonography. 2 nd ed. India, Springer, 2009.

11-BANDI V., LUNN W., ERNST A., et al. Ultrasound versus CT in detecting chest wall invasion by tumor: A prospective study. Chest, 133: 881-886, 2008.

12- GOECKENJAN G., SITTER H., THOMAS M., et al.: Prevention, diagnosis, therapy, and follow-up of lung cancer. Interdisciplinary guideline of the German Respiratory Society and the German Cancer Society-Abridged version. Pneumologie, 65: e51-e75, 2011.

13- GHAYE B. and DONDELINGER R.F.: Imaging guided thoracic interventions. Eur. Respir J., 17: 507-528, 2001.

14- MATHIS G. and GEHMACHER O.: Lung and pleural ultrasound. Schweiz Rundsch Med. Prax, 90: 681-616, 2001.

15- SABRI Y.Y., HAFEZ M.A.F., KAMEL K.M. and ABBAS D.A.: Evaluating the role of ultrasound in chest trauma: Common complications and computed tomography comparative evaluation. EJRNM, 49: 986-992, 2018.

16- NAZERIAN P., VOLPICELLI G., VANNI S., et al.: Accuracy of lung ultrasound for the diagnosis of consolidations when compared to chest computed tomography. AJEM, 33: 620-625, 2015.

17- REISSIG A., HEYNE J.P. and KROEGEL C.: Sonography of lung and pleura in pulmonary embolism' sonomorphologic characterization and comparison with spiral CT scanning. Chest, 1201083: 1977-1983, 2001.

18- XIA Y., YING Y., WANG S., et al.: Effectiveness of lung ultrasonography for diagnosis of pneumonia in adults: A systematic review and meta-analysis. J. Thorac. Dis., 8: 2822-2831, 2016.

19-DUBINSKY T.J., SHAH H., SONNEBORN R. and HIPPE D.S.: Correlation of B-Lines on ultrasonography with interstitial lung disease on chest radiography and CT imaging. Chest, 152: 990-998, 2017.

20- GÖRG C., SEIFART U., GÖRG K. and ZUGMAIER G. Color Doppler sonographic mapping of pulmonary lesions: Evidence of dual arterial supply by spectral analysis. J. Ultrasound Med., 22: 1033-1039, 2003. 


\section{إضافة دورودقة تصوير الصدر \\ بالموجات فوق الصوتية والدوديلر الصوديلر فى آفات الرئة الهحيطية}

$$
\begin{aligned}
& \text { لتعب الموجات فوق الصبتية للصدر وتصوير دوبلر الملون دوراً مهماً فى تشخيص وتوصيف الأمراض } \\
& \text { المختلفة لآفات الرئة المحيطية. } \\
& \text { الهدف من هذه الدراسة هو تقييم دود فحص الصدر بالموجات فوق الصوتية وفحص دويلر في تقيبيم آفات }
\end{aligned}
$$

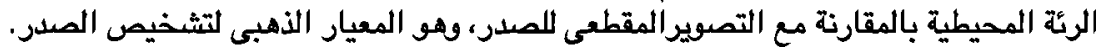

